DAS UMKÄMPFTE BILD 
Ergebnisse der Frauenforschung

Band 34

Herausgegeben im Auftrag des Präsidenten der Freien Universität Berlin von

Prof. Anke Bennholdt-Thomsen, Germanistik

Dr. Ulla Bock, Soziologe

Prof. Marlis Dürkop, Sozialpädagogik

Prof. Ingeborg Falck, Medizin

Prof. Marion Klewitz, Geschichtsdidaktik

Prof. Jutta Limbach, Jura

Prof. Hans Oswald, Pädagogik

Prof. Renate Rott, Soziologie

Dr. Hanna Beate Schöpp-Schilling, Amerikanistik/Anglistik, Germanistik

Koordination: Anita Runge 
Elfi Bettinger

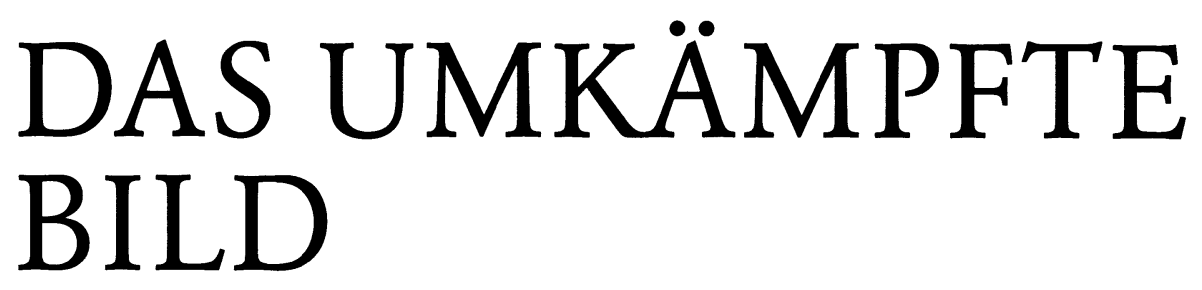

Zur Methapher bei Virginia Woolf

Verlag J. B. Metzler

Stuttgart - Weimar 
Die Deutsche Bibliothek - CIP-Einheitsaufnahme

\section{Bettinger, Elfi:}

Das umkämpfte Bild : zur Metapher bei Virginia Woolf / Elfi Bettinger.

- Stuttgart ; Weimar : Metzler, 1993

(Ergebnisse der Frauenforschung ; Bd. 34)

ISBN 978-3-476-00952-4

NE: GT

ISBN 978-3-476-00952-4

ISBN 978-3-476-03501-1 (eBook)

DOI 10.1007/978-3-476-03501-1

Dieses Werk einschließlich aller seiner Teile ist urheberrechtlich geschützt. Jede Verwertung außerhalb der engen Grenzen des Urheberrechtsgesetzes ist ohne Zustimmung des Verlages unzulässig und strafbar. Das gilt insbesondere für Vervielfältigungen, Übersetzungen, Mikroverfilmungen und die Einspeicherung und Verarbeitung in elektronischen Systemen.

\section{(C) 1993 Springer-Verlag GmbH Deutschland}

Ursprünglich erschienen bei J. B. Metzlersche Verlagsbuchhandlung

und Carl Ernst Poeschel Verlag GmbH in Stuttgart 1993 
Meiner Mutter 


\section{VORWORT}

Nicht nur für literarische, auch für wissenschaftliche Werke gilt, um gleich eine Metapher Virginia Woolfs aufzugreifen, daß sie keine einsamen Einzelgeburten, sondern Ergebnis kollektiver Denkanstrengungen sind. Insbesondere in einem so fruchtbaren Feld wie dem der Woolf-Forschung wird das eigene Denken durch die unterschiedlichen Positionen in durchaus promiskuöser Manier stimuliert. Diese entfalten einen Intertext, vielstimmig und widersprüchlich, der sich nicht einsinnig auflösen und harmonisieren läßt.

Wenn sich das Autorsubjekt nicht mehr als Ursprung, sondern als von vergangenen und gegenwärtigen Diskursen durchkreuzt herausstellt, gerät auch die Textsorte „Vorwort“ unter Druck. Als Ort der Danksagung scheint es in einer heimlich-unheimlichen Beziehung zum proklamierten „Tod des Autors“ zu stehen. Doch da vor dem Vergehen zunächst das Werden gefeiert wird, ist es mir eine Freude, denjenigen danken zu können, die das Entstehen meiner Arbeit in vielfältiger Weise als Väter, Mütter, Geliebte, Hebammen, Freundinnen und Freunde und unterstützende Institutionen gefördert haben.

Mein allererster Dank für wertvolle Unterstützung gilt meinem Doktorvater Prof. Dr. Wilhelm Füger, der diese Arbeit anregte und geduldig die wichtigen Fragen stellte; meiner Zweitgutachterin Prof. Dr. Evelyne Keitel für ihre Ermutigung und nie ermüdende Gesprächsbereitschaft; Friedrich Tontsch für all das, was jenseits des hier Sagbaren liegt. Weiterhin danke ich für Freundschaft, Unterstützung und Anregung Anke Bennholdt-Thomsen, Marie Gebhardt, Elke Hentschel, Heidi Lauper, Franziska Meyer, Manfred Pfister, Helga Quadflieg, Anita Runge, Sobodan Savić und Brunhilde Wehinger. Verschiedene Institutionen haben mich großzügig unterstützt: das Institut für Englische Philologie der Freien Universität Berlin, die vormalige Britische Militärregierung von Berlin und der British Council mit einem Forschungsstipendium, das Jesus College, Cambridge, durch ein Visiting Fellowship sowie der Senat von Berlin, dessen Stipendium im Rahmen des Frauenförderprogramms den Abschluß dieser Arbeit ermöglichte.

Das vorliegende Buch wurde im Sommersemester 1992 an der Freien Universität Berlin als Dissertation angenommen. 
I. EINLEITUNG $\ldots \ldots \ldots \ldots \ldots \ldots \ldots$

1. Virginia Woolf als Mythos . . . . . . . . . . . . . . . 9

2. Eröffnung des Problemhorizonts . . . . . . . . . . . 19

3. Der Betrachtungsgegenstand: Woolfs Bilder . . . . . . . . 22

4. Allgemeiner Forschungsüberblick . . . . . . . . . . . 26

5. Zum Problemfeld der Bilder . . . . . . . . . . . . 34

II. ZUR THEORIE DER METAPHER . . . . . . . . . . . . . . . 43

1. Schwerpunkte der Forschung . . . . . . . . . . . . 43

2. Die Bildfeldtheorie . . . . . . . . . . . . . . . . 49

3. Zu Methode und Darstellung . . . . . . . . . . . . 58

III. DIE BILDFELDER DER REALISTISCHEN TEXTE . . . . . . 63

1. Aufbruch: „Phyllis and Rosamond“ . . . . . . . . . . . . 63

2. Jungfernfahrt: The Voyage Out . . . . . . . . . . . . . . . 72

3. Im Hafen der Ehe: Night and Day . . . . . . . . . . . . . . 99

IV. DIE BILDFELDER DER MODERNISTISCHEN TEXTE . . . . 108

1. Geistesnahrung: Jacob's Room . . . . . . . . . . . . . . 108

2. Im Meer des Lebens: Mrs Dalloway . . . . . . . . . . . . . . . 132

3. Lebensreise und Textgewebe: To the Lighthouse . . . . . . . . . . 153

4. Im Meer der Sprache: The Waves . . . . . . . . . . . . . . . . 179

V. DER DISKURSIVE KONTEXT . . . . . . . . . . . . . . . 206

BIBLIOGRAPHIE . . . . . . . . . . . . . . . . . . . . 242

NAMENVERZEICHNIS . . . . . . . . . . . . . . . . 263 


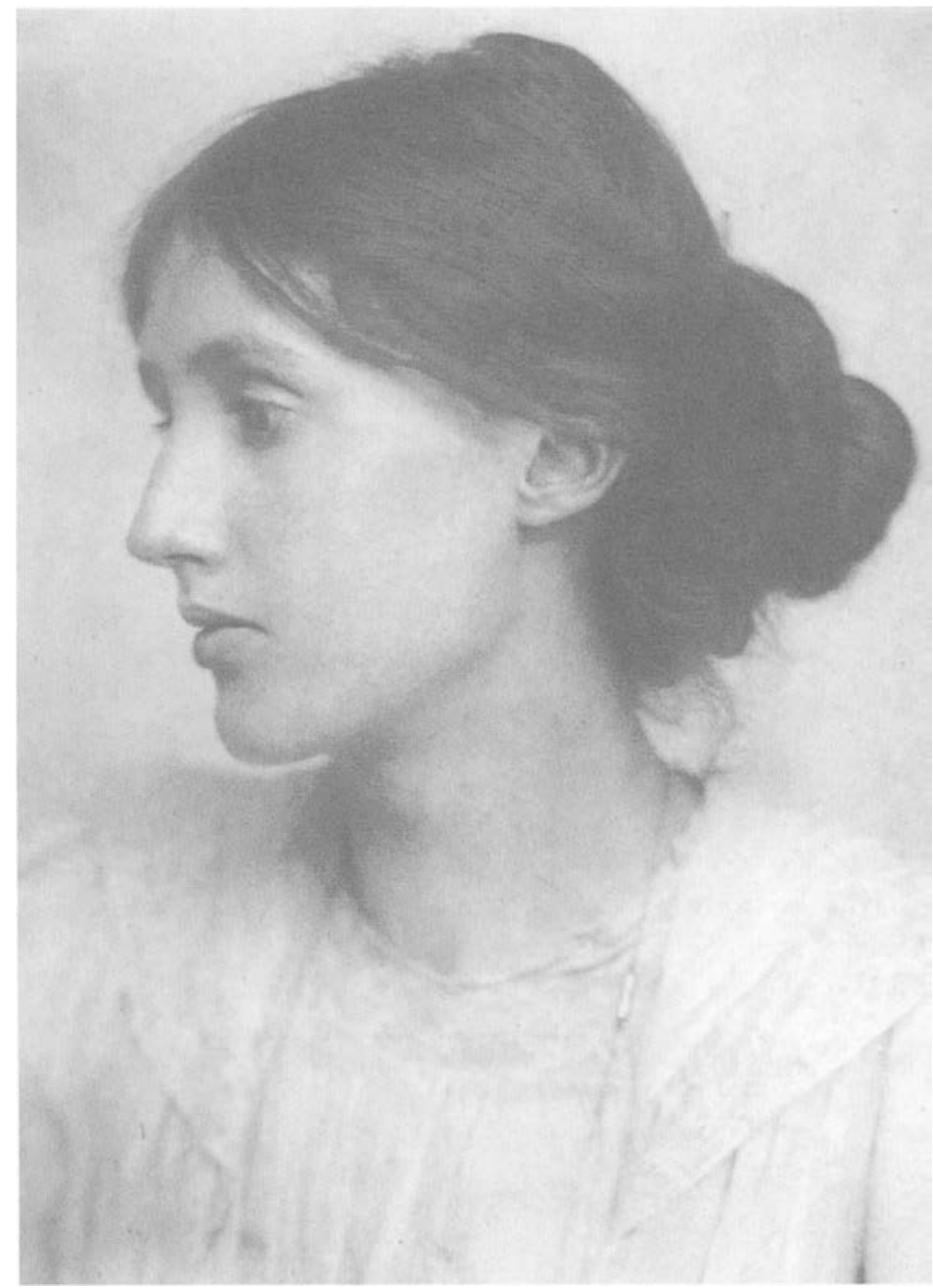

